\title{
Genome-wide association mapping of black point reaction in common wheat (Triticum aestivum L.)
}

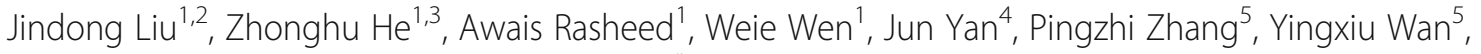
Yong Zhang ${ }^{1}$, Chaojie Xie ${ }^{2}$ and Xianchun Xia ${ }^{1 *}$ (i)

\begin{abstract}
Background: Black point is a serious threat to wheat production and can be managed by host resistance. Marker-assisted selection (MAS) has the potential to accelerate genetic improvement of black point resistance in wheat breeding. We performed a genome-wide association study (GWAS) using the high-density wheat $90 \mathrm{~K}$ and $660 \mathrm{~K}$ single nucleotide polymorphism (SNP) assays to better understand the genetic basis of black point resistance and identify associated molecular markers.

Results: Black point reactions were evaluated in 166 elite wheat cultivars in five environments. Twenty-five unique loci were identified on chromosomes 2A, 2B, 3A, 3B (2), 3D, 4B (2), 5A (3), 5B (3), 6A, 6B, 6D, 7A (5), 7B and 7D (2), respectively, explaining phenotypic variation ranging from 7.9 to $18.0 \%$. The highest number of loci was detected in the A genome (11), followed by the B (10) and D (4) genomes. Among these, 13 were identified in two or more environments. Seven loci coincided with known genes or quantitative trait locus (QTL), whereas the other 18 were potentially novel loci. Linear regression showed a clear dependence of black point scores on the number of favorable alleles, suggesting that QTL pyramiding will be an effective approach to increase resistance. In silico analysis of sequences of resistance-associated SNPs identified 6 genes possibly involved in oxidase, signal transduction and stress resistance as candidate genes involved in black point reaction.

Conclusion: SNP markers significantly associated with black point resistance and accessions with a larger number of resistance alleles can be used to further enhance black point resistance in breeding. This study provides new insights into the genetic architecture of black point reaction.
\end{abstract}

Keywords: 660 K SNP array, 90 K SNP array, Enzymatic browning, Favorable and unfavorable allele, GWAS

\section{Background}

Black point, characterized by dark discoloration at the embryo end of kernels, occurs in most wheat growing regions of the world including China, USA, Australia, Canada and Serbia [1, 2]. It can downgrade end-use quality of the grain due to seed discoloration [3]. Many marketing authorities have regulations on the incidence of black point, such as $\leq 4 \%$ in the USA, $\leq 5 \%$ in Australia, and $\leq 10 \%$ in Canada [4], indicating that grain with black point symptoms is more difficult to market

\footnotetext{
* Correspondence: xiaxianchun@caas.cn

${ }^{1}$ Institute of Crop Sciences, National Wheat Improvement Center, Chinese Academy of Agricultural Sciences (CAAS), 12 Zhongguancun South Street, Beijing 100081, China

Full list of author information is available at the end of the article
}

with consequent economic losses to producers. In addition, black point can decrease the germination percentage and cause impaired seedling development [4]. It can also lead to the presence of toxic secondary metabolites, such as Alternaria mycotoxin and Alternariol monomethyl ether [5-7] that may cause oesophageal cancer [8].

Many studies indicate that black point is enhanced by abiotic stresses, as symptoms more likely occur after exposure to high humidity and extreme temperatures during grain filling $[9,10]$. However, the causes of black point remain unclear and contradictory. Fungi are considered as the causal agents of black point [1]; these include Alternaria alternata [5, 11], Bipolaris sorokiniana [12] and Fusarium proliferatum [6]. However, direct 
association between the presence of fungi and black point development has been discounted by some workers [13-15], who pointed out that it may be caused by enzymatic browning following stress. Oxidases, such as peroxidases (POD) [15, 16], polyphenol oxidase (PPO) $[17,18]$ and lipoxygenase (LOX) [19], that catalyze oxidation of phenolic compounds to brown or black pigments (melanins and quinines) [18, 20], may be triggered by high humidity during the later stages of grain filling. Susceptible varieties have higher POD $[15,21]$ and phenylalanine ammonia-lyase (PAL) (an enzyme involved in phenolic acid biosynthesis) [21] activities.

Although several cultural, biological and chemical control strategies have been used to control black point, breeding resistant cultivars remains the most effective, economic and environmentally sustainable approach to control this disease $[4,22,23]$. Previous studies on the known genetic basis of black point resistance involved classical linkage-mapping methods using bi-parental populations [22-24], in which only two allelic effects can be evaluated for any single locus. Recent advances in genomics, particularly development of the wheat $90 \mathrm{~K}$ [25], $660 \mathrm{~K}$ (JZ Jia, pers. comm.) and $820 \mathrm{~K} \mathrm{SNP}$ arrays [26] have made it feasible to genotype large germplasm collections with high-density SNP markers. As a result, the GWAS based on linkage disequilibrium (LD) has been widely adopted to investigate existing allelic diversity for important and complex agronomic traits. Compared with classical linkage-mapping, GWAS permits a more representative gene pool and a higher mapping resolution, because all historical meiotic events that have occurred in the ancestors of a diverse germplasm panel can be used [27]. Moreover, GWAS bypasses the expense and time of developing mapping populations, and enables the mapping of many traits in one set of genotypes, making the method more efficient and less expensive than linkage mapping [28]. Thus, GWAS has become a powerful alternative approach for linkage mapping [29]. GWAS has been applied to investigate a range of traits, including disease resistance [30,31], end-use quality [32], and yield components [33-35].

The Yellow and Huai River Valleys Facultative Wheat Region is one of the most important agricultural regions of wheat production in China with an area of 15.3 million hectares. Black point has become one of the important diseases in this region due to increased water management and fertilizer use. Breeding for black point resistance could be greatly improved by the identification and use of closely associated molecular markers. Although GWAS has become a powerful approach to dissect the genetic architecture for many traits, it has not been used to analyze traits related to black point. In the present study, we used a diverse panel of 166 elite wheat cultivars in GWAS to (1) dissect the genetic architecture of black point resistance, (2) identify SNPs significantly associated with black point resistance, and (3) search for candidate black point resistance genes for further study.

\section{Results}

\section{Marker coverage and genetic diversity}

A total of 18,920 SNPs from the $90 \mathrm{~K}$ and 283,652 from the $660 \mathrm{~K} \mathrm{SNP}$ array based on the consensus genetic maps and physical map (IWGSC, http://www.wheatgenome.org/) were chosen for GWAS of black point reaction in 166 wheat cultivars (Additional file 1: Table S1). After removing the SNPs with minor allele frequency $(\mathrm{MAF})<5 \% \quad(28,935$ SNPs $)$ and missing data $>20 \%$ (13,715 SNPs), 259,922 SNPs were employed for subsequent analysis (Additional file 2: Table S2). These markers spanned a physical distance of $14,063.9 \mathrm{Mb}$, with an average density of $0.054 \mathrm{Mb}$ per marker. Total of 89,519 (34.4\%), 146,270 (56.3\%) and 24,133 (9.3\%) markers were from the $\mathrm{A}, \mathrm{B}$ and $\mathrm{D}$ genomes, respectively, with corresponding map lengths of 4934.5, 5179.0 and $3950.4 \mathrm{Mb}$. The marker density for the $\mathrm{D}$ genome (0.202 Mb per marker) was lower than that for the A (0.099 Mb per marker) and B (0.042 Mb per marker) genomes. The average genetic diversity and polymorphism information content (PIC) for the whole genome were $0.356(0.009-0.500)$ and $0.285(0.009-0.380)$, respectively. Both the genetic diversity and PIC of the A (0.365 and $0.291)$ and $B(0.363$ and 0.289$)$ genomes were higher than the $\mathrm{D}(0.340$ and 0.265$)$ genome. The number of markers, map length, genetic diversity and PIC for each chromosome are shown in Additional file 2: Table S2.

\section{Population structure and linkage disequilibrium}

In the plot of $\mathrm{K}$ against $\Delta \mathrm{K}$, a break in the slope was observed at $\mathrm{K}=3$ followed by flattening of the curve, indicating that this panel consists of three subgroups, which was consistent with the results of principal components analysis (PCA) and neighbor-joining (NJ) tree analysis (Fig. 1). Subgroup I, the largest group with 62 accessions, was dominated by Shandong and foreign cultivars; Subgroup II consisted of 54 accessions, mainly comprising varieties from Henan, Anhui and Shaanxi provinces; Subgroup III had 50 accessions, most of which were from Henan province (Additional file 1: Table S1).

In total, 12,324 markers from the $90 \mathrm{~K}$ and $660 \mathrm{~K}$ SNP arrays were used to evaluate $\mathrm{LD}$ decay for the whole genome as well as the A, B and D genomes separately. Around $14.3 \%$ of all pairs of loci were in significant LD $(P<0.001)$ with average $r^{2}$ of 0.174 on a genome-wide level by the $90 \mathrm{~K}$ and $660 \mathrm{~K}$ SNP assays. The B genome contained the highest percentage of significant markers (44.2\%), followed by the A (33.6\%) and D (22.2\%) 

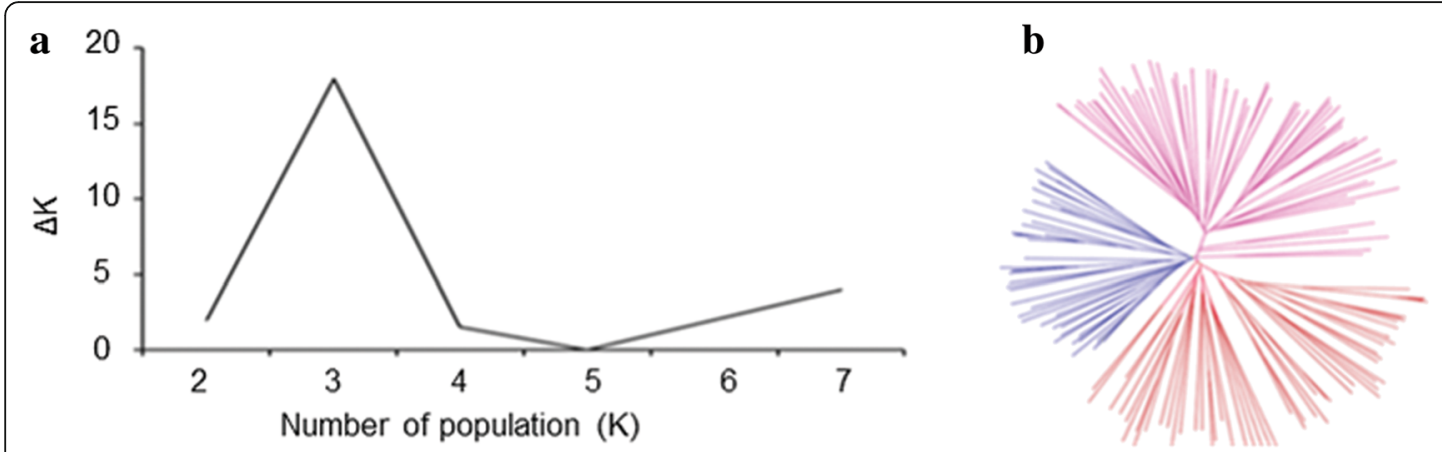

-Subgroup 1

-Subgroup 2

-Subgroup 3
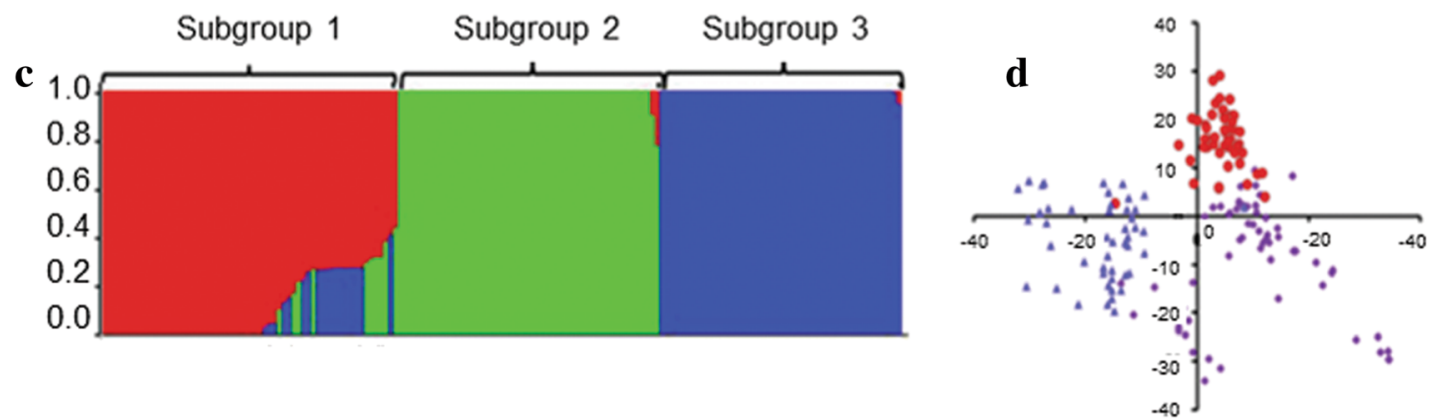

Subgroup 1

'Subgroup 2

'Subgroup 3

Fig. 1 Population structure analysis of 166 wheat accessions. a Estimated $\triangle K$ over five repeats of structure analysis; $\mathbf{b}$ three subgroups inferred by structure analysis; c neighbor-joining (NJ) tree; d principal components analysis (PCA) plots

genomes. The scatter plots of $r^{2}$ against physical distance $(\mathrm{Mb})$ indicated a clear LD decay with increasing physical distance (Additional file 3: Figure S1). According to [28], the critical value for significance of $r^{2}$ was evaluated at $0.079,0.083,0.095$ and 0.082 for the A, B, D and whole genomes, respectively. The point at which the LOESS curve intercepts the critical $r^{2}$ was determined as the average LD decay of the panel [28]. Based on this criterion, $\mathrm{LD}$ decay distance was about $8 \mathrm{Mb}$ for the whole genome. The highest LD decay was observed in the $\mathrm{D}$ genome $(11 \mathrm{Mb})$, followed by the $\mathrm{A}(6 \mathrm{Mb})$ and $\mathrm{B}$ (4 Mb) genomes (Additional file 3: Figure S1).

\section{Phenotypic variations for black point reaction in the field} Continuous variation was observed across five environments (Additional file 4: Figure S2; Additional file 5: Table S3). The resulting best linear unbiased predictors (BLUPs) for black point scores across all environments ranged from 1.6 to $80.6 \%$ with an average of $23.3 \%$ (Additional file 4: Figure S2; Additional file 6: Figure S3), presenting a wide range of reactions for black point and indicating that this diversity panel was ideal for conducting GWAS. Analysis of variance (ANOVA) for black point scores revealed significant differences $(P \leq 0.001)$ among genotypes $(G)$, environments, and genotype $\times$ environment $(\mathrm{G} \times \mathrm{E})$ interactions (Table 1$)$. The broad sense heritability $\left(h^{2}\right)$ estimate for black point scores across all five environments was 0.62, indicating that much of the phenotypic variation was derived from genetic factors and therefore suitable for further association mapping.

\section{Marker-trait association (MTA) analysis}

The MTAs analyzed by the mixed linear model (MLM) in Tassel v5.0 [36] and the FarmCPU [37] were shown in Additional file 7: Table S4 and Additional file 8: Table S5, respectively. Twenty-five loci (221 MTAs) identified by both the Tassel v5.0 and FarmCPU were considered to be more reliable (Table 2, Additional file 9: Table S6); these were distributed on chromosomes $2 \mathrm{~A}, 2 \mathrm{~B}, 3 \mathrm{~A}, 3 \mathrm{~B}$ (2), 3D, 4B (2), 5A (3), 5B (3), 6A, 6B, 6D, 7A (5), 7B and 7D (2), respectively (Table 2, Additional file 9: Table S6), explaining phenotypic variation ranging from 7.9 to $18.0 \%$. Among these loci, 13 on chromosomes 2A, 2B, $3 \mathrm{~A}, 3 \mathrm{~B}(2), 3 \mathrm{D}, 4 \mathrm{~B}, 5 \mathrm{~A}(2), 5 \mathrm{~B}, 7 \mathrm{~A}, 7 \mathrm{~B}$ and $7 \mathrm{D}$ were detected in two or more environments (Table 2, Additional file 9: Table S6). The maximum number of

Table 1 Analysis of variance of black point scores in 166 wheat accessions

\begin{tabular}{llll}
\hline Source of variation & $d f$ & Mean square & $F$ value \\
\hline Replicates (nested in environments) & 9 & 3896 & $7.4^{* * *}$ \\
Environments & 4 & 499,230 & $480.6^{* * *}$ \\
Genotypes & 165 & 5326 & $35.3^{* * *}$ \\
Genotypes $\times$ Environments & 660 & 798 & $4.9^{* * *}$ \\
Error & 1650 & 153 &
\end{tabular}

***significant at $P<0.0001$ 
Table $\mathbf{2}$ Loci for black point resistance in 166 wheat accessions identified by both the Tassel v5.0 and FarmCPU

\begin{tabular}{|c|c|c|c|c|c|c|c|}
\hline Marker $^{\mathrm{a}}$ & Chr ${ }^{b}$ & Physical interval ${ }^{\complement}(b p)$ & Environment $^{d}$ & SNPe & $P$-value ${ }^{f}$ & $R^{29}(\%)$ & QTL/gene ${ }^{\text {h }}$ \\
\hline IWB22408 & $2 \mathrm{~A}$ & $709,831,643-709,831,743$ & E1, E2, E3, E4, E6 & $\mathrm{T} / \underline{\mathrm{C}}$ & $1.2-9.8 \mathrm{E}^{-04}$ & 7.9-14.7 & QBp.caas-2AL [23]; QPPO.caas-2AL [55] \\
\hline PPO-A1 & $2 \mathrm{~A}$ & $712,188,721-712,187,200$ & $E 1, E 2, E 3, E 4, E 6$ & - & $2.4-5.5 E^{-04}$ & $9.9-11.6$ & $P P O-A 1[56]$ \\
\hline AX_108,951,749 & $2 B$ & $714,389,068-714,388,998$ & E2, E3, E4, E6 & $\underline{T} / \mathrm{C}$ & $2.0-7.3 E^{-04}$ & $8.8-11.5$ & QBp.caas-2BL [23] \\
\hline$A X \_111,053,669$ & $3 \mathrm{~A}$ & $9,605,904-9,605,974$ & E2, E3, E4, E6 & $\mathrm{A} / \underline{G}$ & $2.4-8.6 \mathrm{E}^{-04}$ & $8.3-10.4$ & \\
\hline$A X \_108826477$ & 3B & $58,767,930-58,768,000$ & E1, E3 & $\mathrm{A} / \underline{\mathrm{C}}$ & $1.5-9.7 E^{-04}$ & $7.9-11.0$ & \\
\hline AX_108,797,097 & $3 B$ & $695,967,481-695,967,411$ & $\mathrm{E} 1, \mathrm{E} 2, \mathrm{E} 3, \mathrm{E} 6$ & $\mathrm{~A} / \underline{\mathrm{G}}$ & $1.2-9.6 E^{-04}$ & $8.0-11.9$ & QBp.caas-3BL [23] \\
\hline$A X \_110941533$ & $3 \mathrm{D}$ & $4,066,092-4,066,162$ & $E 2, E 3, E 4, E 6$ & $\mathrm{~A} / \underline{\mathrm{C}}$ & $4.1-9.4 \mathrm{E}^{-04}$ & $8.2-9.7$ & \\
\hline AX_108983386 & $4 \mathrm{~B}$ & $6,961,084-6,961,154$ & E5 & $C / \underline{G}$ & $1.7-9.4 \mathrm{E}^{-05}$ & $8.0-10.5$ & \\
\hline$A X \_111488843$ & 4B & $504,944,902-504,944,832$ & $E 1, E 2, E 3, E 6$ & $\mathrm{~A} / \underline{T}$ & $7.0 E^{-06}-2.6 E^{-04}$ & $9.9-15.5$ & \\
\hline IWB8709 & $5 \mathrm{~A}$ & $32,887,598-32,887,698$ & E3 & $\mathrm{A} / \mathrm{G}$ & $1.9-5.3 E^{-04}$ & $8-8.9$ & QBp.caas-5AS [23]; QPod.caas-5AS [57] \\
\hline$A X \_109316564$ & $5 \mathrm{~A}$ & $535,780,381-535,780,311$ & E1, E3, E6 & $\mathrm{T} / \mathrm{G}$ & $4.8-9.9 \mathrm{E}^{-04}$ & $8.1-11.4$ & \\
\hline IWA2223 & $5 A$ & $592,276,555-592,276,708$ & $E 1, E 2, E 3, E 4, E 6$ & $\mathrm{~A} / \underline{G}$ & $3.4 E^{-06}-9.4 E^{-04}$ & $8.0-18.0$ & \\
\hline IWA5214 & $5 B$ & $302,177,272-302,177,428$ & E2 & $\mathrm{A} / \underline{C}$ & $8.60 E^{-04}$ & 8.1 & \\
\hline$A X \_110617778$ & $5 B$ & $531,539,253-531,539,323$ & $E 2, E 3$ & $\mathrm{~A} / \underline{T}$ & $1.4-9.5 E^{-04}$ & $8.0-11.5$ & \\
\hline$A X \_110056162$ & $5 B$ & $556,183,885-556,183,955$ & E5 & $\mathrm{T} / \mathrm{C}$ & $4.2-7.3 E^{-04}$ & $8.3-11.0$ & \\
\hline$A X \_108,821,301$ & $6 \mathrm{~A}$ & $94,2114,60-94,211,390$ & E4 & $C / \underline{G}$ & $1.4-8.0 \mathrm{E}^{-04}$ & $9.1-11.2$ & QBp.caas-6A [23] \\
\hline$A X \_110578177$ & $6 B$ & $676,210,414-676,210,344$ & E1 & $\mathrm{A} / \bar{I}$ & $4.2-5.1 \mathrm{E}^{-04}$ & $8.6-8.9$ & \\
\hline$A X \_109359792$ & $6 \mathrm{D}$ & $217,194,463-217,194,533$ & E4 & $\mathrm{A} / \underline{G}$ & $6.30 \mathrm{E}^{-04}$ & 8.5 & \\
\hline$A X_{-} 111086566$ & $7 \mathrm{~A}$ & $88,862,791-88,862,721$ & E1, E6 & $\mathrm{T} / \underline{\mathrm{C}}$ & $2.2-8.8 E^{-04}$ & $8.6-11.6$ & \\
\hline$A X \_108743156$ & $7 \mathrm{~A}$ & $136,398,412-136,398,482$ & E4, E5, E6 & $\mathrm{A} / \underline{G}$ & $5.0-9.8 E^{-04}$ & 7.9-9.2 & \\
\hline$A X \_109,311,326$ & $7 \mathrm{~A}$ & $609,508,901-609,508,971$ & E2 & $\mathrm{T} / \underline{\mathrm{C}}$ & $8.50 E^{-04}$ & 8.2 & QBp.caas-7AL.2 [23] \\
\hline$A X \_111042346$ & $7 \mathrm{~A}$ & $670,876,731-670,876,661$ & E5, E6 & $C / \underline{G}$ & $2.6-4.9 \mathrm{E}^{-04}$ & $9.0-12.8$ & \\
\hline AX_109491960 & $7 \mathrm{~A}$ & $70,8211,110-708,211,040$ & E4 & $\mathrm{A} / \underline{\mathrm{C}}$ & $1.7-9.1 \mathrm{E}^{-04}$ & $8.4-11.2$ & \\
\hline$A X \_108870509$ & $7 \mathrm{~B}$ & $729,224,017-729,224,087$ & E1, E2, E3, E6 & $\mathrm{A} / \underline{G}$ & $3.0 E^{-05}-8.6 E^{-04}$ & $8.0-13.0$ & \\
\hline AX_109370330 & $7 D$ & $129,917,622-129,917,692$ & E1, E3 & $\mathrm{A} / \underline{\mathrm{C}}$ & $5.2-9.5 \mathrm{E}^{-04}$ & $8.7-9.6$ & \\
\hline$A X \_109033824$ & $7 D$ & $615,826,844-615,826,914$ & E5 & $\mathrm{A} / \underline{\mathrm{C}}$ & $8.30 E^{-04}$ & 8.2 & \\
\hline
\end{tabular}

${ }^{\mathrm{a}}$ Representative markers at the resistance loci

${ }^{\mathrm{b}} \mathrm{Chr}$ : Chromosome

'The physical positions of SNP markers based on wheat genome sequences from the International Wheat Genome Sequencing Consortium (IWGSC, http://www.wheatgenome.org/)

${ }^{d} E 1$ : Anyang 2013; E2: Anyang 2014; E3: Anyang 2015; E4: Suixi 2013; E5: Suixi 2014; E6: Best linear unbiased prediction (BLUP) calculated from all five environments. The data from the results of Tassel v5.0

${ }^{e}$ Favorable allele (SNP) is underlined

${ }^{\mathrm{f}}$ The $P$-values were calculated by the Tassel v5.0

gPercentage of phenotypic variance explained by the MTA from the results of Tassel v5.0

${ }^{\mathrm{h}}$ The previously reported QTL or genes within the same chromosomal regions

loci were found in the A genome (11), followed by the B genome (10), whereas only four loci were identified in the D genome (Table 2; Additional file 9: Table S6).

Kinship-adjusted Manhattan plot summarizing the analysis of black point scores by Tassel v5.0 and FarmCPU are shown in Fig. 2 and Fig. 3, respectively. The quantilequantile $(\mathrm{Q}-\mathrm{Q})$ plot representing expected and observed probabilities of getting associations of SNPs by Tassel v5.0 and FarmCPU are presented in Additional file 10: Figure S4 and Additional file 11: Figure S5, respectively. The LD patterns along 2A, 2B, 3A, 3B, 4B, 5A, 5B, 6A, 6B, 6D, 7A, $7 \mathrm{~B}$ and $7 \mathrm{D}$ can be visualized as heatmaps in Additional file 12: Figure S6.

\section{Relationship between black point reaction and the number of resistance alleles}

To further understand the combined effects of alleles on reaction to black point, we examined the number of favorable alleles in each accession. The numbers of favorable alleles in single accessions ranged from 5 to 21, compared to 4 to 20 unfavorable alleles (Additional file 1: Table S1). The relationships between black point BLUP values and numbers of favorable and unfavorable alleles estimated by linear regression showed a dependence of black point BLUP values on the number of favorable alleles with $r^{2}=0.85$ (Fig. 4a), and number of unfavorable alleles with $r^{2}=0.85$ (Fig. 4b). Thus, accessions with more 


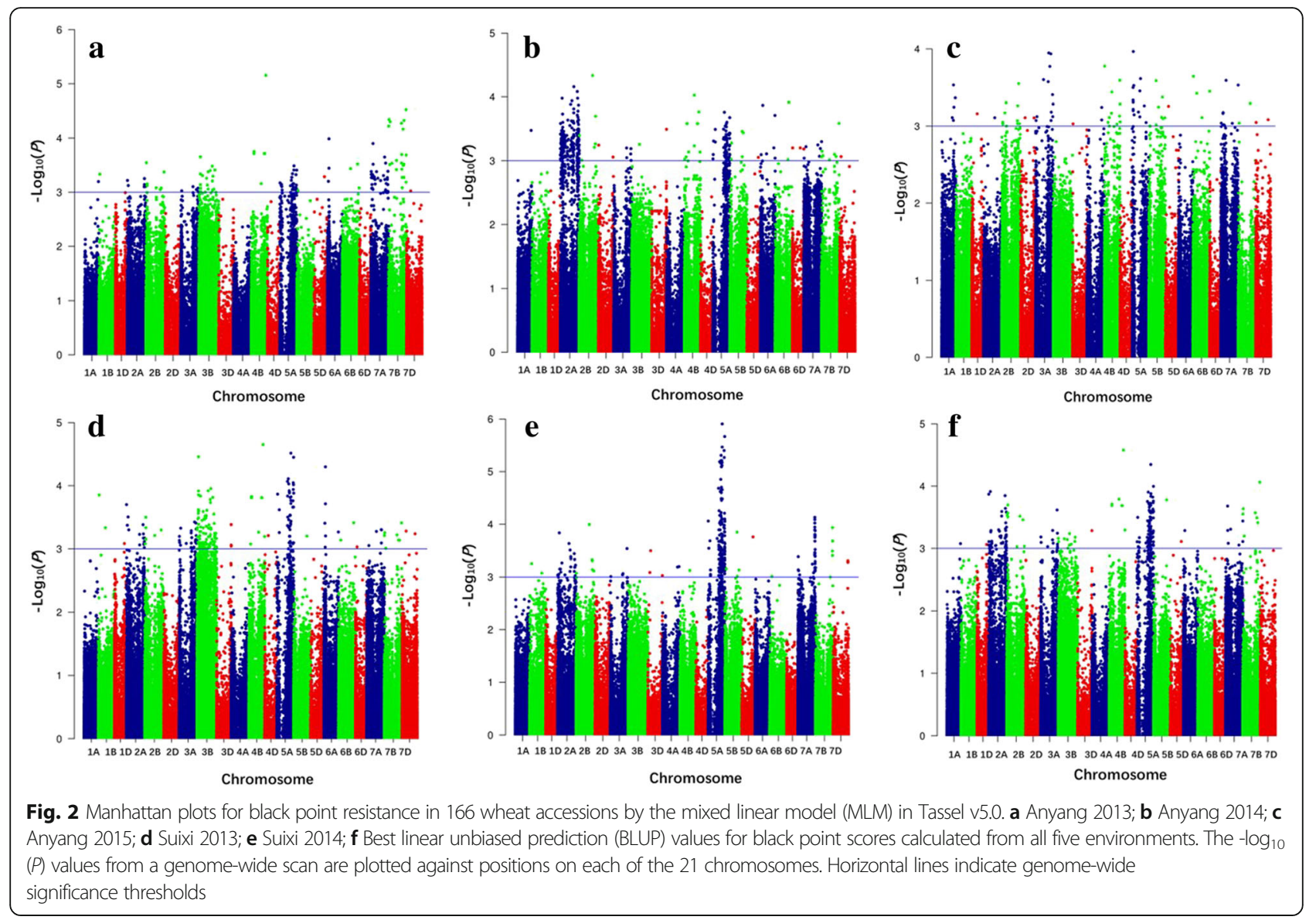

favorable alleles and less unfavorable alleles were more resistant to black point.

\section{Discussion}

The diversity panel, including released cultivars, advanced lines and landraces from different ecological regions, thus had a high genetic diversity with a wide range of reaction to black point. Our data showed that $86.1 \%$ (143) of the 166 accessions were susceptible to black point (black point score $>10 \%$ ), indicating that black point is a considerable threat to wheat production throughout the world. However, most of the previous studies for black point were mainly conducted on pathogen identification, biological characteristics, disease cycle and control $[7,13,38]$. Thus, it is necessary to select cultivars highly resistant to black point and to identify markers significantly associated with resistance to facilitate breeding for resistance by MAS.

\section{Genetic diversity, population structure and linkage disequilibrium}

The mean genetic diversity and PIC of 0.356 and 0.285 , respectively, indicated higher polymorphism than in previous reports $[39,40]$. Our diversity panel thus has high genetic diversity and approximately reflected the genetic diversity in winter wheat from the Yellow and Huai River Valleys Facultative Wheat Region. More than 56\% of SNPs had PIC of 0.20-0.40, which is deemed as a suitable range for GWAS [41]. Furthermore, the A and $B$ genomes had higher genetic diversity and PIC than the D genome, consistent with previous reports [30, 40] (Additional file 2: Table S2). All results indicated that our diversity panel has high genetic diversity and was suitable for GWAS.

The diversity panel could be divided into three subgroups (Fig. 1), and the characterization of the subgroups was largely consistent with geographic origins and pedigrees. For example, Zhongmai 871, Zhongmai 875 and Zhongmai 895, which were derived from Zhoumai 16, clustered with Zhoumai 16 in group 3 (Additional file 1: Table S1). Numerous studies have shown that the lack of appropriate correction for population structure can lead to spurious MTAs [42-45]. Consequently, to eliminate spurious MTAs resulting from population structure, subpopulation data (Q matrix) were considered as fixed-effect factors, whereas the kinship matrix was considered as a random-effect factor, and a MLM implemented in Tassel v5.0 and 


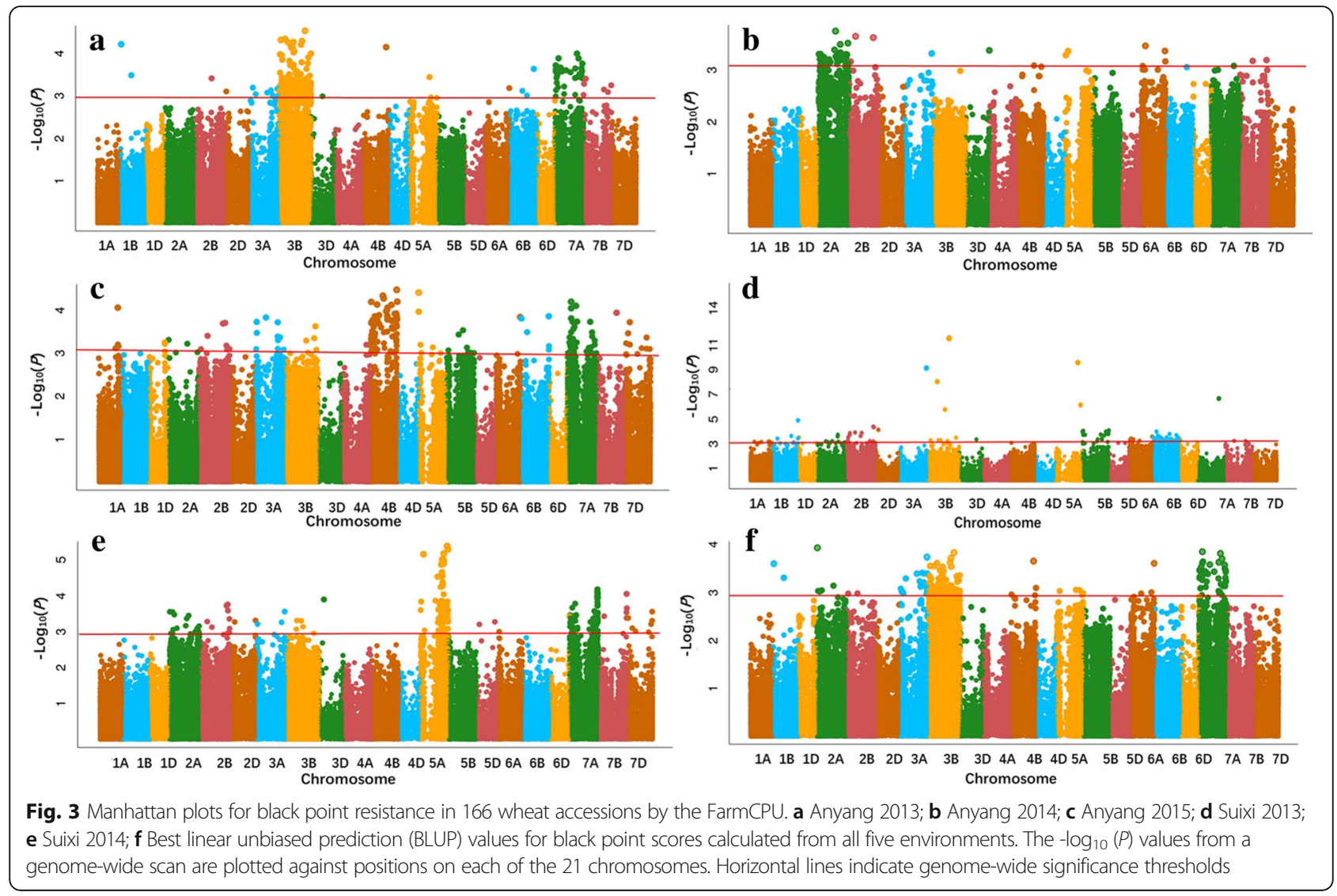

FarmCPU were adopted for association analysis in the current study [36].

The LD decay affects the precision of GWAS and this is influenced by many factors like population structure, allele frequency, recombination rate and selection [44, 46, 47]. Previous studies reported that LD decay in common wheat ranged between 1.5-15 cM using SSR [28, 46, 48], DArT [33] or SNP [30, 47] markers. In this panel, the LD decay was about $8 \mathrm{Mb}$ for the whole genome (Additional file 3: Figure S1), consistent with previous reports. The LD decay of the $\mathrm{D}$ genome $(11 \mathrm{Mb})$ was higher than the $\mathrm{A}(6 \mathrm{Mb})$ and $\mathrm{B}(4 \mathrm{Mb})$ genomes (Additional file 3: Figure S1), also consistent with previous studies [47-49], suggesting that fewer markers are needed for GWAS in the D genome than the $\mathrm{A}$ and $\mathrm{B}$ genomes. The marker densities for the $\mathrm{A}, \mathrm{B}$ and $\mathrm{D}$ genomes were $0.099,0.042$ and $0.202 \mathrm{Mb} /$ marker, and thus highly reliable for detecting MTAs with respect to LD decay in the diversity panel according to Breseghello and Sorrells [28]. The reason for the high LD of the D genome is mainly due to limited infusion of Aegilops tauschii in the evolutionary history of common wheat $[38,49]$. The average $r^{2}(0.174)$ values observed between linked loci pairs were higher than in previous studies [46, 50]. Reif et al. [51] reported that LD $\left(r^{2}\right)$ is expected to be higher in released cultivars than landraces. Moreover, Würschum et al. [52] indicated that QTL with small effect can be detected at higher LD $\left(r^{2}\right)$, whereas only QTL with large effects can be detected at lower LD $\left(r^{2}\right)$. Our results thus suggested a high mapping resolution and strong QTL detection power for black point resistance.

\section{Comparison of the $90 \mathrm{~K}$ and $660 \mathrm{~K}$ SNP arrays}

One of the key factors for GWAS is high marker density in whole genomes because sparse coverage reduces the power of marker identification [53]. Although the $90 \mathrm{~K}$ SNP array has emerged as a promising choice for highdensity, low cost genotyping [34, 54], the presence of large gaps, particularly low coverage for the D genome, reduces the power of marker identification and decreases the precision of QTL mapping. To resolve the problem, the GWAS for black point resistance was performed using 259,922 markers from the $90 \mathrm{~K}$ and $660 \mathrm{~K}$ SNP arrays, providing a greater coverage of the genome. Only 8 loci were identified by the SNPs from $90 \mathrm{~K}$ array, whereas 23 were detected by the $660 \mathrm{~K} \mathrm{SNP}$, indicating that the $660 \mathrm{~K}$ SNP array with its much higher marker density had a significant advantage in GWAS.

\section{Marker-trait associations}

Some black point resistance QTL were previously identified by bi-parental linkage mapping [22, 23], allowing for a comparison between loci identified in the present study 


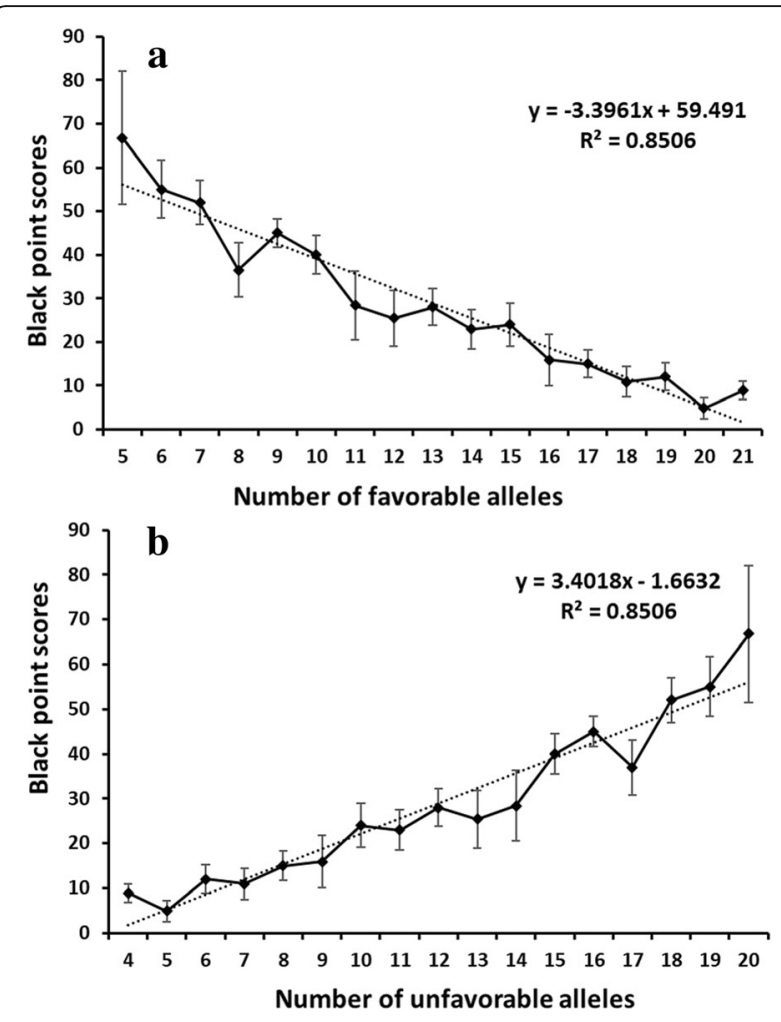

Fig. 4 Linear regression between the number of favorable alleles (a) and unfavorable alleles (b) and the BLUP values for black point scores

and known QTL. Liu et al. [23] found seven stable black point resistance QTL on chromosomes $2 \mathrm{AL}, 2 \mathrm{BL}, 3 \mathrm{BL}$, 5AS, 6A and 7AL (2) in a Linmai 2/Zhong 892 RIL population, which overlapped with loci identified in our study on chromosomes 2AL (IWB22408, $709.8 \mathrm{Mb}), 2 \mathrm{~B}$ (AX108951749, 714.3 Mb), 3BL (AX-108797097, 695.9 Mb), 5AS (IWB8709, 32.8 Mb), 6A (AX-108821301, 94.2 Mb), and 7A (AX-109311326, $609.5 \mathrm{Mb})$ (Tables 2, Additional file 9: Table S6), indicating that GWAS and linkage mapping are complementary in identifying genes. Lehmensiek et al. [22] detected eight black point resistance QTL explaining 4 to $18 \%$ of the phenotypic variation on chromosomes 1D, 2A, 2B, 2D, 3D, 4A, 5A and 7A in Sunco/Tasman and Cascades/AUS1408 doubled haploid (DH) populations by SSR markers. We also identified 11 unique loci in $2 \mathrm{~A}, 2 \mathrm{~B}, 3 \mathrm{D}, 5 \mathrm{~A}$ (3) and 7A (5). The loci on chromosomes 2AL (IWB22408, bin C-2AL1-0.85) and 2BL (AX-108951749, bin 2BL6-0.89-1.00) coincided with the QTL detected by Lehmensiek et al. [22] in chromosomes 2A (Xgwm312, bin C-2AL1-0.85) and 2B (Xgwm319, bin 2BL6-0.89-1.00) (Table 2, Additional file 9: Table S6). However, not all of the QTL detected in linkage analysis were found in GWAS, such as QBp.caas-3AL and QBp.caas-7BS [23]. The reasons for this could be that (a) some QTL may have segregated at low frequency, or not at all in our association panel, and (b) results from the different marker platforms are difficult to align in the absence of complete genome sequences of diverse wheat cultivars.

Oxidases, such as PPO [15] and POD [17], could have enhanced the development of black point. The PPO gene (Ppo-A1) mapped to the long arm of chromosome 2AL in the interval IWB59334-IWBS5777 (706.2-715.3 Mb) [55], overlapped with the loci on chromosome 2AL (IWB22408, $709.8 \mathrm{Mb}$ ) in our study. In addition, the Ppo-A1-specific marker PPO18 [56] was also significantly associated with black point resistance (Table 2). Furthermore, the SNP marker IWA5214 (302.2 Mb) on chromosome 5BL was significantly associated with both black point resistance and PPO activity (Zhai et al. unpublished data). Wei et al. [57] identified a QTL for POD activity on chromosome 5AS (15.9-36.9 Mb) using a RIL population derived from Doumai/Shi 4185, corresponding to the major loci detected on chromosome 5AS (IWB8705, 32.8 Mb) in this study (Additional file 9: Table S6). Shi et al. (unpublished data) identified a locus for POD activity on chromosome 2AL (IWB59334, 715.3 Mb) by GWAS, which overlapped with the locus on chromosome 2AL (IWB22408, 709.8 Mb). Thus, the GWAS results confirmed previous reports implicating phenol metabolism enzymes like PPO and POD in development of black point $[15,17,18]$.

As the genetics of black point reaction are still poorly understood, the remaining 18 loci identified on chromosomes 3A, 3B, 3D, 4B (2), 5A (2), 5B (2), 6B, 6D, 7A (4), $7 \mathrm{~B}$ and $7 \mathrm{D}(2)$ represent potentially new resistance QTL (Table 2); these may contribute to better understand of the architecture of black point reaction and provide more opportunities for resistance breeding. The above results demonstrated that GWAS was a powerful and reliable tool for identification of black point resistance genes.

\section{Candidate genes for black point resistance}

To identify candidate genes for black point resistance, the flanking sequences of SNP markers significantly associated with black point reaction were imported to Blast2Go software, and used as queries to BLAST against the National Center for Biotechnology Information (NCBI) and European Nucleotide Archive (ENA) databases; six candidate genes were identified (Table 3). Bioinformatics analysis indicated that SNP marker $A X$ 111518195 on chromosome 2AL corresponded to peroxisomal biogenesis factor 2, an important gene for biosynthesis of peroxidase, which can accelerate oxidation of phenolic compounds to quinones and is crucial for phenolic metabolism and melanin synthesis $[18,58]$. In addition, the gene-specific marker PPO18 for Ppo-A1 [56] overlapping with the SNP loci on chromosome 2AL was also significantly associated with black point reaction. Fuerst et al. [18] reported that PPO catalyzes oxidation of phenolic compounds to melanins and quinines 
Table 3 Candidate genes for SNPs significantly associated with black point resistance

\begin{tabular}{llllll}
\hline Chromosome & Marker & Candidate gene & Sequence similarity (\%) & Sequence coverage (\%) & Quality parameters \\
\hline 2AL & IWA5463 & F-box repeat & 98 & 98 & $4 \mathrm{E}^{-36}$ \\
2AL & PPO-18 & Polyphenol oxidase (PPO-A1) & - & - & - \\
2AL & AX-111518195 & Peroxisomal biogenesis factor 2 & 97 & 97 & $4 \mathrm{E}^{-12}$ \\
2B & AX-108951749 & Serine/threonine-protein kinase & 97 & 96 & $6 \mathrm{E}^{-06}$ \\
3A & AX-111053669 & Disease resistance RPP8-like protein & 97 & 96 & $1 \mathrm{E}^{-08}$ \\
5AL & IWA2223 & Serine/threonine-protein kinase & 100 & 99 & $8 \mathrm{E}^{-39}$ \\
5A & AX-95684401 & Gibberellin biosynthetic process & 97 & 100 & $4 \mathrm{E}^{-07}$ \\
\hline
\end{tabular}

that may contribute to black point development. Thus, $P p o-A 1$ is a candidate gene for this locus. Marker $A X$ 95684401 on chromosome 5A corresponded to a gibberellin (GA) biosynthetic process protein. GA plays an important role in modulating disease reaction throughout plant development and affects black point development by influencing seed germination [59]. Marker IWA5463 on chromosome 2AL corresponds to an F-box repeat protein, which may affect black point development by regulating signal transduction of gibberellin [59, 60]. F-box proteins have also been implicated in response to various pathogens through targeting substrates in the degradation machinery [61]. Two SNP markers $(A X-108951749$ on $2 \mathrm{~B}$ and IWA2223 on 5AL) encode serine/threonine-protein kinases, which trigger multiple physiological and biochemical reactions in response to abiotic and biotic stresses by mediating perception and transduction of external environmental signals $[62,63]$. We also identified a candidate gene encoding a disease resistance RPP8-like protein $(A X-111053669$ on chromosome $3 \mathrm{~A}$ ), which had been proposed to play an essential role in regulation of responses to a variety of external stimuli, including stress [64]. Bioinformatics analysis of trait-associated SNPs was proven to be an effective tool to find candidate genes for complex agronomic traits [34]. However, black point is a consequence of complicated biological processes and the mechanism of black point formation remains unclear; more detailed experimental analyses are needed to confirm the roles of candidate genes in black point resistance.

\section{Application of MTAs for black point resistance in wheat breeding}

It is difficult to select highly resistant lines at the early stages of a breeding program in the field due to the fact that black point symptoms can be assessed only on mature seed after harvest and are highly affected by environment. A significant additive effect was identified from the linear regression between black point resistance and the number of favorable alleles, indicating that pyramiding of favorable alleles will enhance resistance. Markers significantly associated with complex traits identified by GWAS or QTL mapping can be converted into kompetitive allele-specific PCR (KASP) markers for SNP validation, MAS and QTL fine mapping $[65,66]$. Semi-thermal asymmetric reverse PCR (STARP) also provides a new scalable, flexible and cost-effective approach for using SNP markers in MAS [67]. QTL with consistent effects across multiple environments should be useful for MAS [68]. Thirteen of the 25 loci identified in this study were detected in two or more environments and should be suitable for MAS. Some accessions with higher black point resistance and relatively high number of resistance alleles and excellent agronomic traits, such as Kitanokaori, Norin 67, Yumai 21, Yannong 19, Zhoumai 19, and Zhongmai 871 (Additional file 13: Table S7), should be good parental lines for breeding. Our follow-up studies will focus on validating the effects of these QTL and developing friendly, tightly linked markers that can be used in resistance breeding.

\section{Conclusions}

In the present study, a GWAS for black point resistance in a diversity panel was conducted with the $90 \mathrm{~K}$ and 660 K SNP arrays. Twenty-five resistance loci explained $7.9-18.0 \%$ of the phenotypic variations, demonstrating that GWAS can be used as a powerful and reliable tool for dissecting genes in wheat. The markers significantly associated with black point resistance and the accessions with a higher number of resistance alleles can be used as valuable markers and excellent parent material for resistance breeding. This study improves our understanding of the genetic architecture of black point resistance in common wheat.

\section{Methods}

\section{Plant materials and field trials}

The association panel used in the present study contained 166 diverse cultivars, comprising 144 accessions from the Yellow and Huai River Valley Facultative Wheat Region of China, and 22 accessions from five other countries, including Italy (9), Argentina (7), Japan (4), Australia (1) and Turkey (1) (Additional file 1: Table S1). All accessions were grown at Anyang $\left(35^{\circ} 12^{\prime} \mathrm{N}, 113^{\circ}\right.$ $\left.37^{\prime} \mathrm{E}\right)$ in Henan province during the 2012-2013 and 2013-2014 cropping seasons, and Suixi $\left(33^{\circ} 17^{\prime} \mathrm{N}, 116^{\circ}\right.$ 
23'E) in Anhui province during 2012-2013, 2013-2014 and 2014-2015. Field trials were conducted in randomized complete blocks with three replicates at all locations. Each plot contained three $2 \mathrm{~m}$ rows spaced $20 \mathrm{~cm}$ apart. Agronomic management followed local practices. All wheat accessions are deposited in the National Genebank of China, Chinese Academy of Agricultural Sciences, and available after approval.

\section{Phenotypic evaluation and statistical analysis}

After harvest and threshing three samples of 200 grains were selected from each of the three replicates of each accession, and the percentages of kernels with black point symptoms were determined and averaged. BLUPs across five environments were used as the phenotypic values for association mapping to eliminate environmental effects. BLUP estimation was calculated using the MIXED procedure (PROCMIXED) in SAS v9.3 (SAS Institute, http://www.sas.com).

ANOVA was performed using SAS v9.3 (SAS Institute, http://www.sas.com). Variance components were used to calculate broad sense heritability $\left(h^{2}\right)$ of black point scores as $h^{2}=\sigma_{g}^{2} /\left(\sigma_{g}^{2}+\sigma_{g e}^{2} / r+\sigma_{\varepsilon}^{2} / r e\right)$, where $\sigma_{g}^{2}, \sigma_{g e}^{2}$ and $\sigma_{\varepsilon}^{2}$ represent the genotype, genotype $\times$ environment interaction and residual error variances, respectively, and $e$ and $r$ were the numbers of environments and replicates per environment, respectively.

\section{Genotyping and quality control}

Total genomic DNA for SNP arrays was extracted from five bulked young leaves from each accession using a modified CTAB procedure [69]. The 166 accessions were genotyped using both the Illumina wheat $90 \mathrm{~K}$ SNP (containing 81,587 SNPs) and Affymetrix $660 \mathrm{~K}$ SNP (containing 630,517 SNPs) arrays by Capital Bio Corporation, Beijing, China (http://www.capitalbiotech.com/ ). Accuracy of SNP clustering was validated visually. MAF, genetic diversity and PIC were computed by PowerMarker v3.25 [70] (http://statgen.ncsu.edu/powermarker/). To avoid spurious MTAs, SNP markers with MAF $<0.05$ and missing data $>20 \%$ were excluded from further analyses. The physical positions of SNP markers from the wheat $90 \mathrm{~K}$ and $660 \mathrm{~K} \mathrm{SNP}$ arrays were obtained from the International Wheat Genome Sequencing Consortium website (IWGSC, http://www.wheatgenome.org/), and markers from two SNP arrays were integrated into a common physical map for GWAS.

\section{Population structure}

Population structure was analyzed using 2000 polymorphic SNP markers from the $90 \mathrm{~K}$ and $660 \mathrm{~K}$ SNP arrays with Structure v2.3.4 [41] (http://pritchardlab.stanford.edu/structure.html), which implements a model-based Bayesian cluster analysis. Five independent runs for each $\mathrm{K}$ value from 2 to 12 were performed based on an admixture model. Each run was carried out with 100,000 recorded Markov-Chain iterations and 10,000 burn-in periods. An adhoc quantity statistic $\Delta \mathrm{K}$ based on the rate of change in log probability of data between successive $\mathrm{K}$ values [71] was used to predict the real number of subpopulations. PCA and NJ trees were also used to validate population stratification with the software Tassel v5.0 [44] and PowerMarker v3.25 [70] (http://www.maizegenetics.net).

\section{Linkage disequilibrium}

LD among markers was calculated using the full matrix and sliding window options in Tassel v5.0 with 12,324 evenly distributed SNP markers. The positions of these markers were based on the physical map mentioned above. Pairwise LD was measured using squared allelefrequency correlations $r^{2}$, and significance of pair-wise LD ( $P$-values) was measured by Tassel v5.0 with 1000 permutations. The $r^{2}$ values were plotted against physical distance and a LOESS curve was fitted to the plot to show the association between LD decay and physical map distance. The critical value of $r^{2}$ beyond which the LD was likely to be caused by genetic linkage was determined by taking the 95th percentile in the distribution of $r^{2}$ of the selected loci [28]. The intersection of the fitted curve of $r^{2}$ values with this threshold was considered as the estimate of $\mathrm{LD}$ range.

\section{Genome-wide association analysis}

Associations between genotypic and phenotypic data were analyzed using the kinship matrix in a MLM by Tassel v5.0 to control background variation and eliminate the spurious MTAs. In MLM analysis, the kinship matrix (K matrix) was considered a random-effect factor, whereas the subpopulation data (Q matrix) was considered a fixed-effect factor [43]. The $\mathrm{K}$ matrix was calculated by the software Tassel v5.0 and the Q matrix was inferred by the program Structure v2.3.4. The $P$ value determining whether a SNP marker was associated with the trait and the $R^{2}$ indicating the variation explained by the marker was recorded. The GWAS was also analyzed using the FarmCPU software [37] by $\mathrm{R}$ Language (https://www.r-project.org/). Bonferroni-Holm correction [72] for multiple testing $(\alpha=0.05)$ was too conserved and no significant MTAs were detected with this criterion. Therefore, markers with an adjusted $-\log _{10}(P$-value $) \geq 3.0$ were regarded as significant markers for black point reaction [73-75], as shown in Manhattan plots using the ggplot2 code in $\mathrm{R}$ Language. Important $P$ value distributions (observed $P$ values plotted against expected $P$ values) were shown in $\mathrm{Q}-\mathrm{Q}$ plots.

We checked the LD $\left(r^{2}\right)$ among markers significantly associated with black point reaction on the same chromosomes to compare the resistance loci. LD block 
on the same chromosome were computed and visualized by Haploview v4.2 [76] (www.broadinstitute.org/haploview/ haploview). To compare resistance loci identified in the present study with known genes/QTL, deletion bin information for SSR and SNP markers was obtained following [23].

\section{The effect of favorable alleles on black point resistance}

Each locus comprises two alleles based on SNP marker a single base substitution, transition or transversion. Alleles with positive effects leading to higher black point resistance are referred to as "favorable alleles", and those leading to lower resistance are "unfavorable alleles". The representative SNPs at the resistance loci were used to count the frequencies of favorable and unfavorable alleles and their allelic effects were determined (Table 2). Regression analysis between favorable, unfavorable alleles and black point scores were conducted using the line chart function in Microsoft Excel 2016.

\section{In silico annotation of SNPs}

To identify candidate genes or putative protein functions of SNP flanking-regions, the flanking sequences corresponding to the SNP markers significantly associated with black point resistance were used in BLASTn and BLASTx searches against ENA (http://www.ebi.ac.uk/ena) and NCBI (http://www.ncbi.nlm.nih.gov/) databases. Sequences were imported to Blast2Go software (https://www.blast2go.com/) in fasta formats that were blasted, mapped and annotated using standard parameters embedded in the software.

\section{Additional files}

Additional file 1: Table S1. The 166 wheat accessions used in the genome-wide association study (GWAS) for black point reaction and their origins. (DOCX $27 \mathrm{~kb}$ )

Additional file 2: Table S2. Basic statistical analysis of SNP markers used for genome-wide association study (GWAS) of 166 wheat accessions. (DOCX $19 \mathrm{~kb}$ )

Additional file 3: Figure S1. LD decay estimated in 166 wheat accessions based on 12,324 markers from the $90 \mathrm{~K}$ and $660 \mathrm{~K}$ SNP arrays. (DOCX $97 \mathrm{~kb}$ )

Additional file 4: Figure S2. Black point scores evaluated for 166 wheat accessions. Reported values are the best linear unbiased predictions (BLUP) value for black point scores across five environments. (DOCX $430 \mathrm{~kb}$ )

Additional file 5: Table S3. The black point scores of the 166 wheat accessions across the five environments. (XLSX $25 \mathrm{~kb}$ )

Additional file 6: Figure S3. Frequency distributions for black point scores for 166 wheat accessions in five environments. (a) Anyang 2013; (b) Anyang 2014; (c) Anyang 2015; (d) Suixi 2013; (e) Suixi 2014; (f) Best linear unbiased predictions (BLUP) value for black point scores across five environments. (DOCX $292 \mathrm{~kb}$ )

Additional file 7: Table S4. Marker-trait associations (MTAs) for black point reaction in 166 wheat accessions analyzed by the mixed linear model (MLM) in Tassel v5.0. (XLSX $80 \mathrm{~kb}$ )

Additional file 8: Table S5. Marker-trait associations (MTAs) for black point resistance in 166 wheat accessions analyzed by the FarmCPU. (XLSX $94 \mathrm{~kb})$
Additional file 9: Table S6. Marker-trait associations (MTAs) for black point resistance in 166 wheat accessions identified by both the Tassel v5.0 and FarmCPU. (XLSX $18 \mathrm{~kb})$

Additional file 10: Figure S4. Quantile-quantile (Q-Q) plot for black point scores in 166 wheat accessions by the mixed linear model (MLM) in Tassel v5.0. (a) Anyang 2013; (b) Anyang 2014; (c) Anyang 2015; (d) Suixi 2013; (e) Suixi 2014; (f) Best linear unbiased predictions (BLUP) value for black point scores across five environments. (DOCX $682 \mathrm{~kb}$ )

Additional file 11: Figure S5. Quantile-quantile (Q-Q) plot for black point scores in 166 wheat accessions by the FarmCPU. (a) Anyang 2013; (b) Anyang 2014; (c) Anyang 2015; (d) Suixi 2013; (e) Suixi 2014; (f) Best linear unbiased predictions (BLUP) value for black point scores across five environments. (DOCX $379 \mathrm{~kb}$ )

Additional file 12: Figure S6. $L D$ heatmap of all wheat chromosomes showing extent of pairwise linkage disequilibrium between SNP markers significantly associated with black point reactions. (DOCX $4561 \mathrm{~kb}$ )

Additional file 13: Table S7. Accessions with high black point resistance. (DOCX $17 \mathrm{~kb})$

\section{Abbreviations}

ANOVA: Analysis of variance; BLUPs: Resulting best linear unbiased predictors; DH: Doubled haploid; GA: Gibberellin; GWAS: Genome-wide association study; KASP: Kompetitive allele-specific PCR; LD: Linkage disequilibrium; LOX: Lipoxygenase; MAF: Minor allele frequency; MAS: Marker-assisted selection; MLM: Mixed linear model; MTA: Marker-trait association; NJ: Neighbor-joining; PAL: Phenylalanine ammonia-lyase; PCA: Principal components analysis; PIC: Polymorphism information content;

POD: Peroxidase; PPO: Polyphenol oxidase; Q-Q: Quantile-quantile; QTL: Quantitative trait loci (locus); RIL: Recombinant inbred line; SNP: Single nucleotide polymorphism; SSR: Simple sequence repeat; STARP: Semi-thermal asymmetric reverse PCR

\section{Acknowledgments}

We thank Prof. R. A. McIntosh, at Plant Breeding Institute, University of Sydney, for reviewing this manuscript.

\section{Funding}

This work was funded by the National Key Research and Development Program of China (2016YFD0101802, 2016YFE0108600, 2014BAD01B05), National Natural Science Foundation of China (31461143021), Gene Transformation Project (2016ZX08009-003) and CAAS Science and Technology Innovation Program.

\section{Availability of data and materials}

The phenotypic data of the current study is available in the Additional file 5 :

Table S3. Any other datasets used and/or analyzed are available upon request.

\section{Authors' contributions}

$J D L$ performed the experiment and wrote the paper. WEW, JY, PZZ, YXW and YZ participated in field trials. ZHH, AR, CJX and XCX designed the experiment and assisted in writing the paper. All authors have read and approved the final version of this manuscript.

Ethics approval and consent to participate

We declare that these experiments comply with the ethical standards and legislations in China, and all wheat varieties were collected in accordance with national guidelines.

Consent for publication

Not applicable.

Competing interests

The authors declare that they have no competing interests.

\section{Publisher's Note}

Springer Nature remains neutral with regard to jurisdictional claims in published maps and institutional affiliations. 


\section{Author details}

'Institute of Crop Sciences, National Wheat Improvement Center, Chinese Academy of Agricultural Sciences (CAAS), 12 Zhongguancun South Street, Beijing 100081, China. ${ }^{2}$ Department of Plant Genetics \& Breeding/State Key Laboratory for Agrobiotechnology, China Agricultural University, 2 Yuanmingyuan West Road, Beijing 100193, China. ${ }^{3}$ International Maize and Wheat Improvement Center (CIMMYT) China Office, c/o CAAS, 12 Zhongguancun South Street, Beijing 100081, China. ${ }^{4}$ Institute of Cotton Research, Chinese Academy of Agricultural Sciences (CAAS), 38 Huanghe Street, Anyang, Henan 455000, China. ${ }^{5}$ Crop Research Institute, Anhui Academy of Agricultural Sciences, 40 Nongke South Street, Hefei, Anhui 230001, China

Received: 28 May 2017 Accepted: 10 November 2017 Published online: 23 November 2017

\section{References}

1. Conner RL, Davidson JGN. Resistance in wheat to black point caused by Alternaria alternata and Cochliobolus sativus. Can J Plant Pathol. 1988:68:351-9.

2. Sissons M, Sissons S, Egan N. The black point status of selected tetraploid species and Australian durum wheat and breeding lines. Crop Sci. 2010;50:1279-86.

3. Dexter JE, Matsuo RR. Effect of smudge and black point, mildewed kernels, and ergot on durum wheat quality. Cereal Chem. 1982;59:63-9.

4. Li QY, Qin Z, Jiang YM, Shen CC, Duan ZB, Niu JS. Screening wheat genotypes for resistance to black point and the effects of diseased kernels on seed germination. J Plant Dis Protect. 2014;121:79-88.

5. Logrieco A, Bottalico A, Mulé G, Moretti A, Perrone G. Epidemiology of toxigenic fungi and their associated mycotoxins for some Mediterranean crops. Eur J Plant Pathol. 2003;109:645-67.

6. Desjardins AE, Busman M, Proctor RH, Stessman R. Wheat kernel black point and fumonisin contamination by Fusarium proliferatum. Food Addit Contam. 2007;24:1131-7.

7. Busman M, Desjardins AE, Proctor RH. Analysis of fumonisin contamination and the presence of Fusarium in wheat with kernel black point disease in the United States. Food Addit Contam. 2012;29:1092-100.

8. Palacios SA, Susca A, Haidukowski M, Stea G, Cendoya E, Ramírez ML, Chulze SN, Farnochi MC, Moretti A, Torres AM. Genetic variability and fumonisin production by Fusarium proliferatum isolated from durum wheat grains in Argentina. Int J Food Microbiol. 2015;201:35-41.

9. Maloy OC, Spetch KL. Black point of irrigated wheat in Central Washington. Plant Dis. 1988;72:1031-3.

10. Fernandez MR, Wang $H$, Singh AK. Impact of seed discoloration on emergence and early plant growth of durum wheat at different soil gravimetric water contents. Can J Plant Pathol. 2014;36:509-16.

11. Southwell RJ, Brown JF, Wong PT. Effect of inoculum density, stage of plant growth and dew period on the incidence of black point caused by Alternaria alternata in durum wheat. Ann Appl Biol. 1980;96:29-35.

12. Kumar J, Schäfer $P$, Hückelhoven $R$, Langen $G$, Baltruschat $H$, Stein $E$ Nagarajan S, Kogel KH. Bipolaris sorokiniana, a cereal pathogen of global concern: cytological and molecular approaches towards better control. Mol Plant Pathol. 2002;3:85-195.

13. Williamson PM. Black point of wheat: in vitro production of symptoms, enzymes involved, and association with Alternaria alternata. Aust J Agri Res. 1997:48:13-9.

14. Hudec K. Pathogenicity of fungi associated with wheat and barley seedling emergence and fungicide efficacy of seed treatment. Biologia. 2007:62:287-91.

15. March TJ, Able J, Schultz C, Able AJA. Novel late embryogenesis abundant protein and peroxidase associated with black point in barley grains. Proteomics. 2007;7:3800-8

16. Mak Y, Willows RD, Roberts TH, Wrigley CW, Sharp PJ, Copeland LES. Black point is associated with reduced levels of stress, disease and defence related proteins in wheat grain. Mol Plant Pathol. 2006;7:177-89.

17. Anderson JV, Morris CF. An improved whole-seed assay for screening wheat germplasm for polyphenol oxidase activity. Crop Sci. 2001;41:1697-705.

18. Fuerst EP, Okubara PA, Anderson JV, Morris CF. Polyphenol oxidase as a biochemical seed defense mechanism. Front Plant Sci. 2014;5:689.

19. Porta H, Rocha-Sosa M. Plant lipoxygenases. Physiological and molecular features. Plant Physiol. 2002;130:15-21.
20. Tomás-Barberán FA, Espín JC. Phenolic compounds and related enzymes as determinants of quality in fruits and vegetables. J Sci Food Agr. 2001;81:853-76.

21. Regnier T, Macheix JJ. Changes in wall bound phenolic acids, phenylalanine and tyrosine ammonia-lyases, and peroxidases in developing durum wheat grains (Triticum turgidum L. Var. durum). J Agric Food Chem 1996;44: 1727-1730.

22. Lehmensiek A, Campbell AW, Williamson PM, Michalowitz M, Sutherland MW, Daggard GQTL. For black point resistance in wheat and the identification of potential markers for use in breeding programs. Plant Breed. 2004;123:410-6.

23. Liu JD, He ZH, Wu L, Bai B, Wen WE, Xie CJ, Xia XC. Genome-wide linkage mapping of QTL for black point reaction in bread wheat (Triticum aestivum L.). Theor Appl Genet 2016;129:2179-2190.

24. March TJ, Able JA, Willsmore K, Schultz CJ, Able AJ. Comparative mapping of a QTL controlling black point formation in barley. Funct Plant Biol. 2008; 35:427-37.

25. Wang S, Wong D, Forrest K, Allen A, Chao S, Huang BE, Maccaferri M, Salvi S, Milner SG, Cattivelli L, Mastrangelo AM, Stephen S, Barker G, Wieseke R, Plieske J, International Wheat Genome Sequencing Consortium, Lillemo M, Mather D, Appels R, Dulferos R, Brown-Guedira G, Korol A, Akhunova AR, Feuillet C, Salse J, Morgante M, Pozniak C, Luo MC, Dvorak J, Morell M, Dubcovsky J, Ganal M, Tuberosa R, Lawley C, Mikoulitch I, Cavanagh C, Edwards KJ, Hayden M, Akhunov E. Characterization of polyploid wheat genomic diversity using the high-density 90,000 SNP array. Plant Biotech J. 2014:12:787-96.

26. Winfield MO, Allen AM, Burridge AJ, Barker GL, Benbow HR, Wilkinson PA, Coghill J. High-density SNP genotyping array for hexaploid wheat and its secondary and tertiary gene pool. Plant Biotechnol J. 2015;14:1195-206.

27. Flint-Garcia SA, Thornsberry JM, Buckler ES. Structure of linkage disequilibrium in plants. Annu Rev Plant Biol. 2003;54:357-74.

28. Breseghello F, Sorrells ME. Association mapping of kernel size and milling quality in wheat (Triticum aestivum L.) cultivars. Genetics. 2006;172:1165-77.

29. Zhu CS, Gore M, Buckler ES, Status YJM. Prospects of association mapping in plants. Plant Genome. 2008;1:5-20.

30. Zegeye H, Rasheed A, Makdis F, Badebo A, Ogbonnaya FC. Genome-wide association mapping for seedling and adult plant resistance to stripe rust in synthetic hexaploid wheat. PLoS One. 2014;9:e105593.

31. Pasam RK, Bansal U, Daetwyler HD, Forrest KL, Wong D, Petkowski J, Willey N. Detection and validation of genomic regions associated with resistance to rust diseases in a worldwide hexaploid wheat landrace collection using BayesR and mixed linear model approaches. Theor Appl Genet. 2017;130:777-93.

32. Marcotuli I, Houston K, Waugh R, Fincher GB, Burton RA, Blanco A, Gadaleta A. Genome-wide association mapping for arabinoxylan content in a collection of tetraploid wheats. PLoS One. 2015;10:e0132787.

33. Rasheed A, Xia XC, Ogbonnaya F, Mahmood T, Zhang Z, Mujeeb-Kazi A, He $\mathrm{ZH}$. Genome-wide association for grain morphology in synthetic hexaploid wheats using digital imaging analysis. BMC Plant Biol. 2014;14:128.

34. Ain QU, Rasheed A, Anwar A, Mahmood T, Imtiaz M, Mahmood T, Xia XC, He ZH, Quraishi UM. Genome-wide association for grain yield under rain-fed conditions in historical wheat cultivars from Pakistan. Front Plant Sci. 2015;6:743.

35. Sun CW, Zhang FY, Yan XF, Zhang XF, Dong ZD, Cui DQ, Chen F. Genome-wide association study for 13 agronomic traits reveals distribution of superior alleles in bread wheat from the yellow and Huai Valley of China. Plant Biotechnol J. 2017; doi:10.1111/pbi.12690.

36. Bradbury PJ, Zhang Z, Kroon DE, Casstevens TM, Ramdoss Y, Buckler ESTASSEL. Software for association mapping of complex traits in diverse samples. Bioinformatics. 2007;23:2633-5.

37. Liu X, Huang M, Fan B, Buckler ES, Zhang Z. Iterative usage of fixed and random effect models for powerful and efficient genome-wide association studies. PLoS Genet. 2016;12(2):e1005767.

38. Kahl SM, Ulrich A, Kirichenko AA, Müller ME. Phenotypic and phylogenetic segregation of Alternaria infectoria from small-spored Alternaria species isolated from wheat in Germany and Russia. J Appl Microbiol. 2015;119:1637-50.

39. Chen XJ, Min DH, Tauqeer AY, Genetic HYG. Diversity, population structure and linkage disequilibrium in elite Chinese winter wheat investigated with SSR markers. PLoS One. 2012;7:e44510.

40. Lopes M, Dreisigacker S, Peña R, Sukumaran S, Reynolds M. Genetic characterization of the wheat association mapping initiative (WAMI) panel for dissection of complex traits in spring wheat. Theor Appl Genet. 2014; 128:453-64. 
41. Botstein D, WIllte RL, Skolinck M. Construction of a genetic linkage map in man using restriction fragment length polymorphisms. Am J Hum Genet. 1980;32:314-9.

42. Chao S, Zhang W, Dubcovsky J, Sorrells M. Evaluation of genetic diversity and genome-wide linkage disequilibrium among US wheat (Triticum aestivum L.) germplasm representing different market classes. Crop Sci. 2007:47:1018-30.

43. Pritchard JK, Stephens M, Rosenberg NA, Donnelly P. Association mapping in structured populations. Am J Hum Genet. 2000;67:170-81.

44. Yu J, Buckler ES. Genetic association mapping and genome organization of maize. Curr Opin Biotechnol. 2006;17:155-60.

45. Cormier F, Gouis JL, Dubreuil P, Lafarge S, Praud S. A genome-wide identification of chromosomal regions determining nitrogen use efficiency components in wheat (Triticum aestivum L.). Theor Appl Genet 2014;127:2679-2693.

46. Hao CY, Wang LF, Ge HM, Dong YC, Zhang XY. Genetic diversity and linkage disequilibrium in Chinese bread wheat (Triticum aestivum L.) revealed by SSR markers. PLoS One. 2011;6:e17279.

47. Sukumaran S, Dreisigacker S, Lopes M, Chavez P, Reynolds MP. Genome-wide association study for grain yield and related traits in an elite spring wheat population grown in temperate irrigated environments. Theor Appl Genet. 2015;128:353-63.

48. Chao S, Dubcovsky J, Dvorak J, Luo MC, Baenziger SP, Matnyazov R, Clark DR, Talbert LE, Anderson JA, Dreisigacker S, Glover K, Chen J, Campbell K, Bruckner PL, Rudd JC, Haley S, Carver BF, Perry S, Sorrells ME, Akhunov ED. Population and genome specific patterns of linkage disequilibrium and SNP variation in spring and winter wheat (Triticum aestivum L.). BMC Genomics 2010;11:727.

49. Edae EA, Byrne PF, Haley SD, Lopes MS, Reynolds MP. Genome-wide association mapping of yield and yield components of spring wheat under contrasting moisture regimes. Theor Appl Genet. 2014;127:791-807.

50. Chen GF, Zhang H, Deng ZY, Wu RG., Li DM, Wang MY, Tian JC. Genome-wide association study for kernel weight-related traits using SNPs in a Chinese winter wheat population. Euphytica 2016;212:173-185.

51. Reif JC, Maurer HP, Korzun V, Ebmeyer E, Miedaner T, Würschum T, Mapping QTL. With main and epistatic effects underlying grain yield and heading time in soft winter wheat. Theor Appl Genet. 2011;123:283-2927.

52. Würschum T, Maurer HP, Kraft T, Janssen G, Nilsson C, Reif JC. Genome-wide association mapping of agronomic traits in sugar beet. Theor Appl Genet. 2011;123:1121-31

53. Poznial CJ, Clarke JM, Clarke FR. Potential for detection of marker-trait associations in durum wheat using unbalanced, historical phenotypic datasets. Mol Breed. 2012;30:1537-50.

54. Allen AM, Barker GLA, Berry ST, Coghill JA, Gwilliam R, Kirby S, Robinson P, Brenchley RC, D'Amore R, McKenzie N, Waite D, Hall A, Bevan M, Hall N, Edwards KJ. Transcript-specific, single-nucleotide polymorphism discovery and linkage analysis in hexaploid bread wheat (Triticum aestivum L.). Plant Biotechnol J 2011;9:1086-1099.

55. Zhai SN, He ZH, Wen WE, Jin H, Liu JD, Zhang Y, Liu ZY, Xia XC. Genome-wide linkage mapping of flour color-related traits and polyphenol oxidase activity in common wheat. Theor Appl Genet. 2016;129:377-94.

56. Sun DJ, He ZH, Xia XC, Zhang LP, Morris CF, Appels R, Ma WJ, Wang HA, Novel STS. Marker for polyphenol oxidase activity in bread wheat. Mol Breed. 2005;16:209-18.

57. Wei JX, Geng HW, Zhang Y, Liu JD, Wen WE, Xia XC, Chen XM, He ZH. Mapping quantitative trait loci for peroxidase activity and developing gene-specific markers for TaPod-A1 on wheat chromosome 3AL. Theor Appl Genet. 2015;128:2067-76.

58. Kumar S, Kawałek A, van der Klei IJ. Peroxisomal quality control mechanisms. Curr Opin Microbiol. 2014;22:30-7.

59. Sun TP, Gubler F. Molecular mechanism of gibberellin signaling in plants. Annu Rev Plant Biol. 2004;55:197-223.

60. Frigerio M, Alabadí D, Pérez-Gómez J, García-Cárcel L, Phillips AL, Hedden P, Blázquez MA. Transcriptional regulation of gibberellin metabolism genes by auxin signaling in Arabidopsis. Plant Physiol. 2006;142:553-63.

61. Kim HS, Delaney TP. Arabidopsis SON1 is an F-box protein that regulates a novel induced defense response independent of both salicylic acid and systemic acquired resistance. Plant Cell. 2002;14:1469-82.

62. Xiong $L$, Yang $Y$. Disease resistance and abiotic stress tolerance in rice are inversely modulated by an abscisic acid-inducible mitogen-activated protein kinase. Plant Cell. 2003;15:745-59.
63. Li FH, FL F, Sha LN, He L, Li WC. Differential expression of serine/threonine protein phosphatase type- $2 \mathrm{C}$ under drought stress in maize. Plant Mol Bio Rep. 2009;27:29-37.

64. Hameed U, Pan YB, labal J. Genetic analysis of resistance gene analogues from a sugarcane cultivar resistant to red rot disease. J Phytopathol. 2015; 163:755-63.

65. Semagn K, Babu R, Hearne S, Olsen M. Single nucleotide polymorphism genotyping using Kompetitive allele specific PCR (KASP): overview of the technology and its application in crop improvement. Mol Breed 2014;33:1-14.

66. Rasheed A, Hao YF, Xia XC, Khan A, Yb X, Varshney RK, He ZH. Crop breeding chips and genotyping platforms: progress, challenges, and perspectives. Mol Plant. 2017;10:1047-64.

67. Long YM, Chao WS, Ma GJ, SS X, Qi LL. An innovative SNP genotyping method adapting to multiple platforms and throughputs. Theor Appl Genet. 2016:130:597-607.

68. Veldboom LR, Lee M. Genetic mapping of quantitative trait loci in maize in stress and non-stress environments: I. Grain yield and yield components. Crop Sci. 1996;36:1310-9.

69. Doyle JJ, Doyle JL. A rapid DNA isolation procedure from small quantities of fresh leaf tissues. Phytochem Bull. 1987;19:11-5.

70. Liu K, Muse SV. PowerMarker. An integrated analysis environment for genetic marker analysis. Bioinformatics. 2005:21:2128-9.

71. Evanno G, Regnaut S, Goudet J. Detecting the number of clusters of individuals using the software STRUCTURE: a simulation study. Mol Ecol. 2005;14:2611-20.

72. Holm SA. Simple sequentially rejective multiple test procedure. Scand J Stat. 1979;6:65-70

73. Houston K, Russell J, Schreiber M, Halpin C, Oakey H, Washington JM, Booth A, Shirley N, Burton RA, Fincher GB, Waugh R. A Genome wide association scan for $(1,3 ; 1,4)$ - $\beta$-glucan content in the grain of contemporary 2 - row spring and winter barleys. BMC Genomics 2014;15:907.

74. Gurung S, Mamidi S, Bonman JM, Xiong M, Brown-Guedira G, Adhikari TB. Genome-wide association study reveals novel quantitative trait loci associated with resistance to multiple leaf spot diseases of spring wheat. PLoS One. 2014;9:e108179.

75. Bellucci A, Torp AM, Bruun S, Magid J, Andersen SB, Rasmussen SK. Association mapping in scandinavian winter wheat for yield, plant height, and traits important for second-generation bioethanol production. Front Plant Sci. 2015;6:1046.

76. Barrett JC, Fry B, Maller J, Daly MJ. Haploview: analysis and visualization of LD and haplotype maps. Bioinformatics. 2005;21:263-5.

\section{Submit your next manuscript to BioMed Central and we will help you at every step:}

- We accept pre-submission inquiries

- Our selector tool helps you to find the most relevant journal

- We provide round the clock customer support

- Convenient online submission

- Thorough peer review

- Inclusion in PubMed and all major indexing services

- Maximum visibility for your research

Submit your manuscript at www.biomedcentral.com/submit 